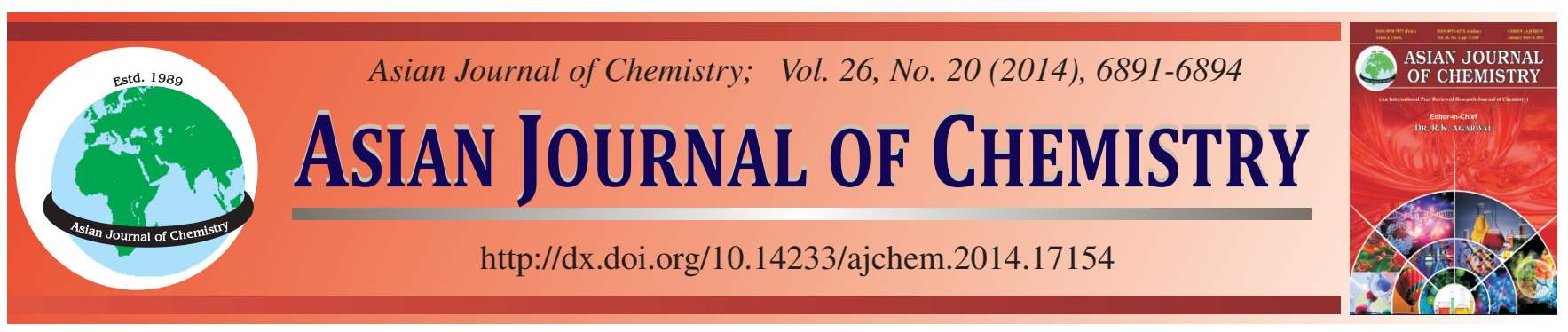

\title{
Effect of Molecular Structure on the Adsorption of Soluble Aromatic Hydrocarbons by Activated Carbon
}

\author{
S.M. YAKOUT ${ }^{1,2, *}$ \\ ${ }^{1}$ Biochemistry Department, College of Science, King Saud University, PO Box, 2455, Riyadh, 11451, Kingdom of Saudi Arabia \\ ${ }^{2}$ Atomic Energy Authority, Hot Laboratories Centre, 13759, Egypt \\ *Corresponding author: E-mail: sobhy.yakout@gmail.com
}

\begin{abstract}
The compounds known as polycyclic aromatic hydrocarbons (PAHs) and benzene, toluene, ethylbenzene and xylene (BTEX) are soluble aromatic hydrocarbons among the most carcinogenic, mutagenic and toxic found in aquatic systems. The effect of adsorbate properties (molecular structure, molecular weight and solubility) on adsorption from liquid phase was studied for the chosen organic substances of aromatic character using rice husk activated carbon (RHAC). The possible mechanisms of adsorption process and solute interactions with carbon surface were analyzed. The effect of substituent in aromatic ring of adsorbate molecule on the adsorption affinity toward carbon surface was regarded. Adsorption increases with decreasing solubility of adsorbent and increases with the molecular weight of the solute. Adsorption rate correlated with number of methyl group in the molecule as well as the length of the side chain.
\end{abstract}

Keywords: Adsorption, Soluble aromatic hydrocarbons, BTEX, Polycyclic aromatic hydrocarbon, Activated carbon.

\section{INTRODUCTION}

Both polycyclic aromatic hydrocarbon (PAH) and benzene, toluene, ethylbenzene and xylene (BTEX) are widespread environmental pollutants. This group of compounds attained public attention in the last decades. There are several sources of these compounds in the environment, the main one being associated with spills involving the release of petroleum products like gasoline, diesel fuel, and lubricants, among others ${ }^{1}$. Benzene, toluene, ethylbenzene and xylene and polycyclic aromatic hydrocarbons exhibit toxic and carcinogenic properties. The US EPA has classified them as priority pollutants ${ }^{2,3}$. These compounds are toxic to both the environment and to human health, since they can act as central nervous system depressants and even exhibit chronic toxicity. Thus, any contamination from these sources deserves attention, not only by direct contact (such as inhalation of vapors) but also from the presence of these compounds in water bodies used for human consumption ${ }^{4}$. In addition, polycyclic aromatic hydrocarbon compounds also can be find in several other matrices, such as alcohol drinks ${ }^{5}$, instant coffee ${ }^{6}$, $\operatorname{wood}^{7}$, food $^{8-10}$, peats ${ }^{11}$, canned bivalves $^{12}$ and soil ${ }^{13}$.

Benzene, toluene, ethylbenzene and xylene are relatively water-soluble as compared to polycyclic aromatic hydrocarbons, a property that enables them to migrate along the subsurface contaminating drinking water sources ${ }^{14}$. However, polycyclic aromatic hydrocarbons are characterized by low water solubility, except for smaller molecules like naphthalene and a high affinity for association with organic matter in soil and water ${ }^{15}$. They are environmentally persistent due to their relative chemical stability and resistance to biodegradation.

Activated carbon in different forms (powder, granular, or brittle) has been employed over the years in the treatment of both industrial and municipal waste water due to its ability to adsorb a wide variety of both organic and inorganic compounds to non detectable trace levels. Among all remediation technologies for treating water contaminated with benzene, toluene, ethylbenzene and xylene and polycyclic aromatic hydrocarbons, adsorption by activated carbon appears to be the most cost-effective, energy efficient and environmentally sound approach $^{16-18}$. However, there are no reports concerning effect of the molecular structure on the adsorption of soluble aromatic hydrocarbon by activated carbon.

With this study, we aim to determine the adsorption of two representatives of a functionally different soluble aromatic hydrocarbon class (benzene, toluene, ethylbenzene and xylene and polycyclic aromatic hydrocarbon) to active carbon. Our central goal was to develop a mechanistic understanding of the interplay between the molecular characteristics of sorbate and sorbent as a determinant of aromatic hydrocarbon sorption. 
EXPERIMENTAL

Rice husk activated carbon (RHAC) was prepared according procedures in our previous work ${ }^{16} .40 \mathrm{~g}$ of powdered rice husk was impregnated with $100 \mathrm{~mL}$ of $40 \%$ phosphoricacid. This mass was heated gradually up to $700{ }^{\circ} \mathrm{C}$ with in $2 \mathrm{~h}$ in a vertical cylindrical furnace and soaked at this temperature for $3 \mathrm{~h}$. After cooling the carbonized mass was washed several times with bidistilled water until $\mathrm{pH} 6.5$ and dried at $110^{\circ} \mathrm{C}$. Elemental analysis of sorbent materials was performed by CHNEl emental Analyzer (Perkin-Elmer, Norwach, USA). The BET-surface area of rice husk activated carbon was calculated from $\mathrm{N}_{2}$-gas adsorption that was obtained by NOVA 3200; V 6.05 gas sorption analyzer, Quantachrome, U.S.A. Elemental analysis and physical characterization concerned with pore volume and surface area of rice husk activated carbonare listed in Table-1.

TABLE-1

PHYSICAL AND CHEMICAL CHARACTERIZATION R.h ACTIVATED CARBON

\begin{tabular}{lccc}
\hline Inner BET surface area $\left(\mathrm{m}^{2} / \mathrm{g}\right)$ & 446 & $\mathrm{pH}$ & 2.1 \\
\hline Total pore volume $\left(\mathrm{cm}^{3} / \mathrm{g}\right)$ & 0.301 & $\mathrm{C} \%$ & 58.5 \\
Mean pore radius $\left(\mathrm{A}^{\circ}\right)$ & 15.5 & $\mathrm{H} \%$ & 3.4 \\
Micro pore volume $\left(\mathrm{cm}^{3} / \mathrm{g}\right)$ & 0.196 & $\mathrm{~N} \%$ & 2.9 \\
Density $(\mathrm{g} / \mathrm{mL})$ & 0.447 & $\mathrm{Cl} \%$ & 1.9 \\
Water content $($ wt. \%) & 9 & $\mathrm{~S} \%$ & 2.1 \\
& & Ash & 3.8 \\
\hline
\end{tabular}

Employed benzene, toluene, ethylbenzene and xylene compounds including benzene (B), toluene (T), ethyl benzene (E), p-xylene (X) and employed polycyclic aromatic hydrocarbon compounds including naphthalene (NA), phenanthrene $(\mathrm{PH})$ and pyrene $(\mathrm{PY})$ were analytical gradewith $>99 \%$ purity and purchased from Merck. A stocksolution in methanol for benzene, toluene, ethylbenzene and xylene and acetone for polycyclic aromatic hydrocarbon compounds was prepared. The solutions used in the experiments and in the construction of analytical curves were prepared immediately before use. Studied compounds concentration were determined by gas chromatography (model: HP-6890) with flame ionization detector (PT-GC-FID). Purge and Trap (model:HP-7695) was used as sample preparationin case of benzene, toluene, ethylbenzene and xylene analysis. However split-splitless injector was used in case of polycyclic aromatic hydrocarbon analysis.

Adsorption experiments were carried out in $25 \mathrm{~mL}$ glass vials with gas-tight caps, non-permeable by organic vapors. About $10 \mathrm{~mL}$ aliquots of each component of benzene, toluene, ethylbenzene and xylene and polycyclic aromatic hydrocarbon with an initial concentration of $10 \mathrm{mg} / \mathrm{L}$ were shaken with 10 $\mathrm{mg}$ of rice husk activated carbon each mixture was filtered andequilibrium concentration was measured after equilibrium time of 2 days. Sorption capacity of adsorbent was calculated by:

$$
\mathrm{q}_{\mathrm{e}}^{\text {ads }}=\mathrm{V}\left(\mathrm{C}_{\mathrm{o}}-\mathrm{C}_{\mathrm{e}}\right) / \mathrm{ms}
$$

where $\mathrm{C}_{0}$ and $\mathrm{C}_{\mathrm{e}}$ are the initial and equilibrium concentration $(\mathrm{mg} / \mathrm{L})$, respectively, mis the mass of dry carbon sample used $(\mathrm{g})$ and vis the volume of solution $(\mathrm{mL})$. Adsorption rate constant, $\mathrm{K}$ were determined by plotting amount adsorbed $(\mathrm{mg} / \mathrm{g})$ at time $\mathrm{t} ; \mathrm{q}_{\mathrm{t}}$ versus the square root of time $\left(\mathrm{t}^{0.5}\right)$ using Weber-Morris eqn. $2^{19}$ :

$$
\mathrm{q}_{\mathrm{t}}=\mathrm{k} \mathrm{t} \mathrm{t}^{0.5}
$$

\section{RESULTS AND DISCUSSION}

Benzene, toluene, ethylbenzene and xylene and polycyclic aromatic hydrocarbon are hydrocarbons with specific recipes and shapes (Fig. 1 and 2). Benzene, toluene, ethylbenzene and xylene have benzene ring bonded to chains, However polycyclic aromatic hydrocarbon have more than one benzene ring. These recipes and shapes can cause concern in the environment. These compounds are considered volatile, meaning that they have a relatively low boiling point and molecular weight as shown in Table-2. Benzene, toluene, ethylbenzene and xylene are relatively water-soluble as compared to polycyclic aromatic hydrocarbons, a property that enables them to migrate along the subsurface contaminating drinking water sources ${ }^{14}$. However, polycyclic aromatic hydrocarbons are characterized by low water solubility, except for smaller molecules like naphthalene and a high affinity for association with organic matter in soil and water ${ }^{15}$. They are environmentally persistent due to their relative chemical stability and resistance to biodegradation.

Adsorption studies: In one of our recent studies ${ }^{17,18}$. The amount of benzene, toluene, ethylbenzene and xylene and polycyclic aromatic hydrocarbon sorbed on rice husk activated carbon was found to be greatly affected by contact time.

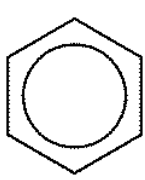

Benzene

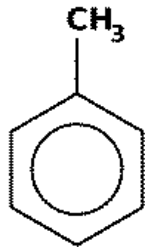

Toluene

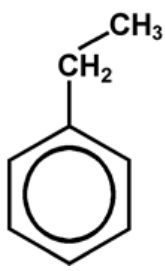

Ethyl benzene

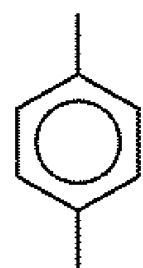

P-xylene

TABLE-2

PHYSICOCHEMICAL PROPERTIES OF STUDIED BTEX AND POLYCYCLIC AROMATIC HYDROCARBON COMPOUNDS

\begin{tabular}{llccc}
\hline \multicolumn{1}{c}{ Compound } & m.f. & m.w. & b.p. $\left({ }^{\circ} \mathrm{C}\right)$ & Water solubility $(\mathrm{mg} / \mathrm{L})$ \\
\hline Benzene & $\mathrm{C}_{6} \mathrm{H}_{6}$ & 78.11 & 80.1 & 1750 \\
Toluene & $\mathrm{C}_{7} \mathrm{H}_{8}$ & 92.14 & 110.6 & 535 \\
Ethyl benzene & $\mathrm{C}_{8} \mathrm{H}_{10}$ & 106.17 & 136.2 & 2.13 \\
P-xylene & $\mathrm{C}_{8} \mathrm{H}_{10}$ & 106.17 & 138.4 & 2.69 \\
Naphthalene & $\mathrm{C}_{10} \mathrm{H}_{18}$ & 128.17 & 218 & 152 \\
Phenanthrene & $\mathrm{C}_{10} \mathrm{H}_{10}$ & 178.23 & 340 & 31.7 \\
Pyrene & $\mathrm{C}_{16} \mathrm{H}_{10}$ & 202.26 & 404 & 3.15 \\
\hline
\end{tabular}




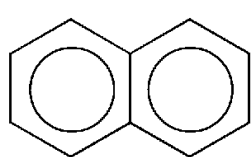

Naphthalene

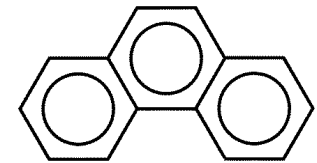

Phenanthrene

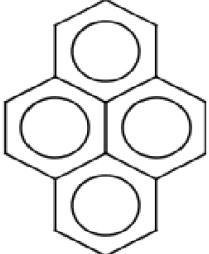

Pyrene
Fig 2. Polycyclic aromatic hydrocarbon compounds

Adsorption capacity increased with increasing the shaking time until reaching saturation where the amount sorbed attains a constant value. The saturation level is specific and depends on the adsorbate, nature of the adsorbent and the chemical composition of medium. Maximum adsorption was observed after two days ( $48 \mathrm{~h}$ ) behind, which there is no further, increases in the adsorption. Hence, $48 \mathrm{~h}$ shaking time was considered to be sufficient for the adsorption of benzene, toluene, ethylbenzene and xylene and polycyclic aromatic hydrocarbon compounds on rice husk activated carbon and was used for all subsequent experiments. Adsorption rate constant K, was determined from the slopes of the amount sorbed (q) $v s . \mathrm{t}^{0.5}$ according Weber-Morris eqn. 2. The evaluated sorption rates, $\mathrm{Kp}$ are given in Table-3.

\begin{tabular}{ccc}
\multicolumn{3}{c}{ TABLE-3 } \\
\multicolumn{3}{c}{ ADSORPTION RATES OF BTEX AND PAH } \\
\hline Compound & $\mathrm{K},\left(\mathrm{mg} / \mathrm{g} \mathrm{h}^{0.5}\right) \times 10^{-3}$ & $\mathrm{~K},\left(\mathrm{mmol} / \mathrm{g} \mathrm{h}^{0.5}\right) \times 10^{-3}$ \\
\hline \multicolumn{3}{c}{$\mathrm{BTEX:}$} \\
\hline Benzene & 20.0 & 0.26 \\
Toluene & 34.0 & 0.37 \\
Ethylbenzene & 40.1 & 0.38 \\
$p$-xylene & 88.7 & 0.84 \\
\hline \multicolumn{3}{c}{ PAH: } \\
\hline Naphthalene & 101.5 & 0.8 \\
Phenanthrene & 156 & 0.88 \\
Pyrene & 200 & 0.99 \\
\hline
\end{tabular}

It is clear that the molecular configuration has a significant influence on the adsorption rate, According Fig. 3 a remarkable dependence could be exist between the observed $\mathrm{K}$ values, the adsorption rate and the molecular weight. $\mathrm{K}$ values for the various components increase as the molecular weight increases as in the following order: $\mathrm{B}<\mathrm{T}<\mathrm{E}<\mathrm{X}<\mathrm{NA}<\mathrm{Ph}<\mathrm{Py}$. As the number of methyl group attached to the aromatic ring increases, the adsorption rate increases (Fig. 4). In order to consider the effect of aromatic solute substituent on adsorption mechanism it is necessary to eliminate the differences in hydrophobicity by normalization to solutes solubility ${ }^{20}$. In the case of all solutes we found almost the same course of adsorption. It means that the solute hydrophobicity is the driving force in adsorption of the investigated aromatics from the aqueous solutions. Also, as the chain length increases (from $p$-xylene to ethylbenzene), the adsorption rate increases. Such data indicate that, the polycyclic aromatic hydrocarbon adsorption rate is greater than that of benzene, toluene, ethylbenzene and xylene; this can be explained by the fact that as the number of aromatic rings increases the removal capacity increases (Fig. 5) i.e. the influence of aromatic ring in case of polycyclic aromatic hydrocarbon on the removal of eachsolute is greater than that

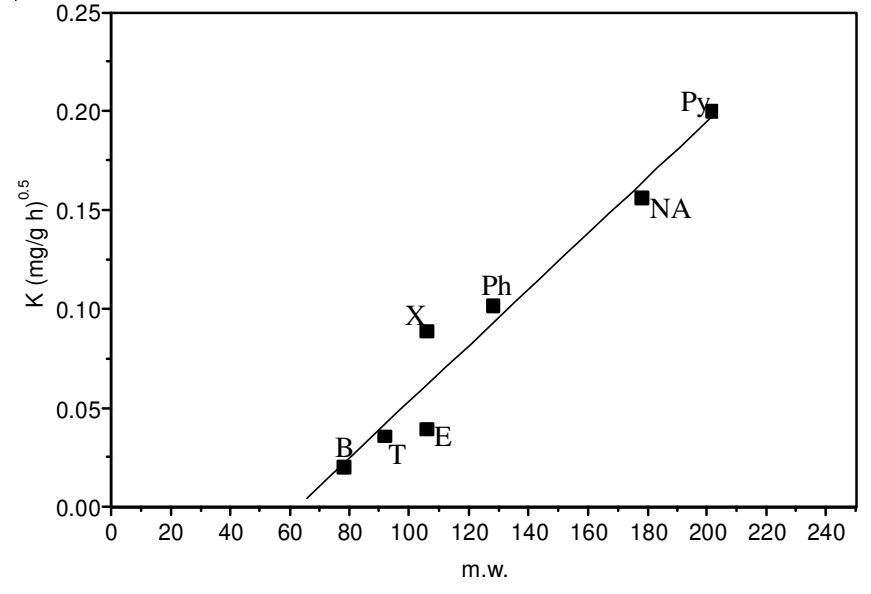

Fig. 3. Effect of molecular weight on the adsorption rate

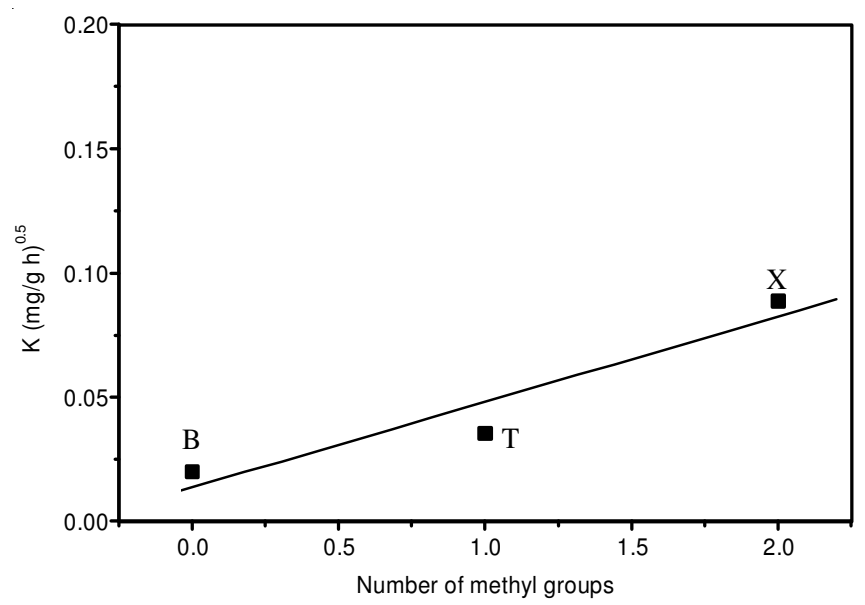

Fig. 4. Relationship between the methyl groups and adsorption rate

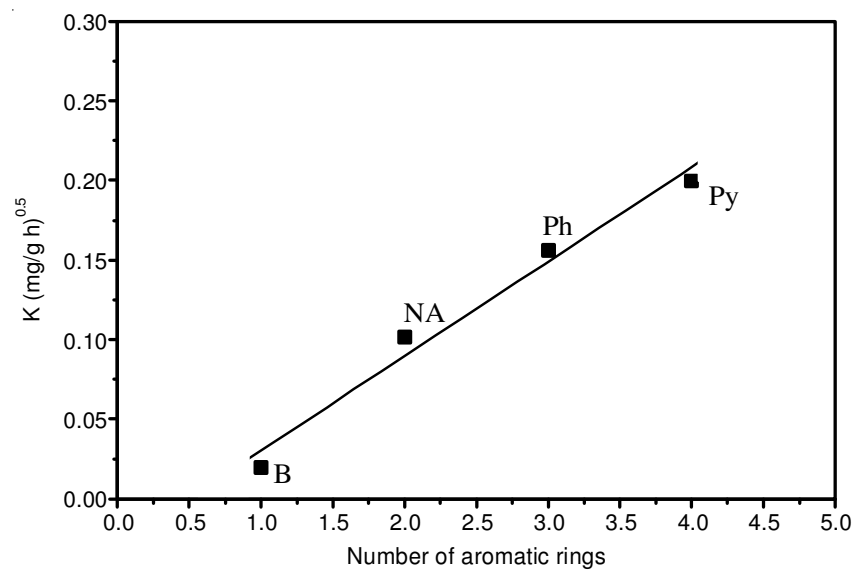

Fig. 5. Relationship between aromatic rings and the adsorption rate

of methyl group in case of benzene, toluene, ethylbenzene and xylene.

When the amount of solute adsorbed is expressed in molar units instead of weight units as in column (b) in Table-2, the $\mathrm{K}$ values for the various components have a similar order as above. This can be explained by the fact that not only the molecular weight or the side chain has the predominant effect on the adsorption rate but also there are some factors should be considered e.g. solubility in water. The solubility order: B 
$>\mathrm{T}>\mathrm{E}>\mathrm{X}>\mathrm{NA}>\mathrm{Ph}>\mathrm{Py}$ and the adsorption rate has the reverse order: $\mathrm{B}<\mathrm{T}<\mathrm{E}<\mathrm{X}<\mathrm{NA}<\mathrm{Ph}<\mathrm{Py}$ i.e. as the solubility increases, the adsorption rate decreases (Fig. 6).

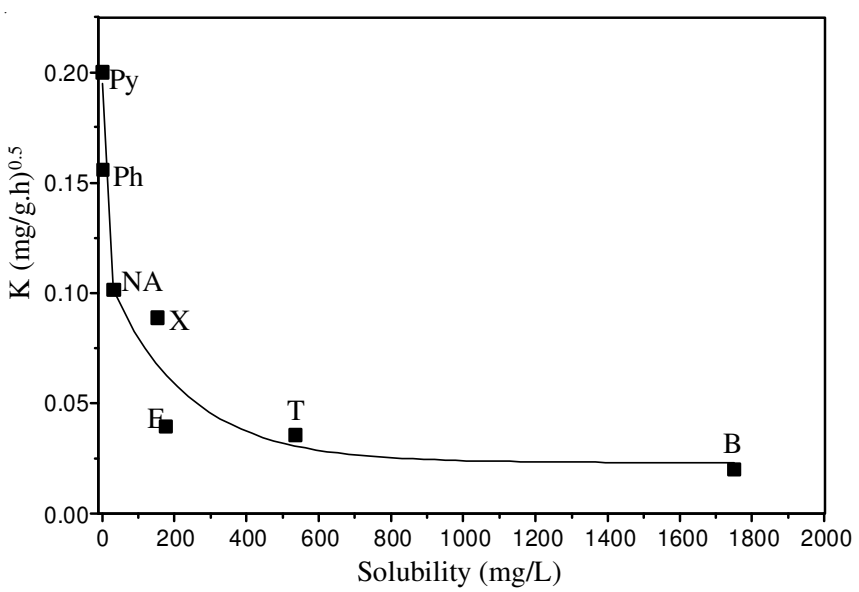

Fig. 6. Effect of solubility on adsorption rate

The molecular structure of an adsorbate is important in determining the degree of adsorption that actually occurs. In general, the adsorbability of a compound increases with increasing molecular weight and increasing number of functional groups such as double bonds or halogens ${ }^{21}$. Branched chain compounds are more sorbable than straight-chain compounds and molecules that are low in polarity and solubility tend to be preferentially adsorbed. If the screening action of the carbon pore is effective, large molecules are more sorbable than small molecules of similar chemical nature.

The degree of solubility of the solute is also of primary concern for adsorption. There is an inverse relationship between the extent of adsorption of a particular solute and its solubility in the solvent from which the adsorption occurs. High solubility means that the solute-solvent bonds are stronger than the attractive forces between the solute and the adsorbent ${ }^{22}$.

Adsorption mechanism: Thus, adsorption of the studied aromatic molecules on carbonaceous materials is a result of the non-electrostatic interactions between the solute molecular forms and sorbent surface, i.e., the dispersion interactions between the $\pi$-electrons of the solute aromatic ring and the graphene layers of adsorbent. the solute hydrophobicity determined by its solubility, is the main important factor in ad sorption mechanism. However, the properties of substituent in solute aromatic benzene ring has to be also taken into account. Depending on their character they can withdraw or release electrons affecting the dispersion interactions between the solute aromatic ring and the carbon graphene layers. The functional group being the electron donor activates the benzene ring or graphene plane by pushing the electrons towards it and creating a partially negative charge. This increasing their adsorption capacity $p$-xylene than toluene than benzene.

\section{Conclusion}

The adsorption behaviour of souble aromatic hydrocarbons including benzene, toluene, ethylbenzene and xylene and polycyclic aromatic hydrocarbon compounds onto rice husk based carbonhas been examined. Adsorption rates affected by different factors such as M.Wt; molecular configuration, structural of the alkyl group attached to the benzene ring and solubility. The differences in adsorptionuptakes for studied adsorbates were mainly a result of the difference in their hydrophobicity and molecular structure.

\section{ACKNOWLEDGEMENTS}

This project was supported by King Saud University, Deanship of Scientific Research, College of Science Research Centre.

\section{REFERENCES}

1. A. El Nemr and A.M. Abd-Allah, Chemosphere, 52, 1711 (2003).

2. B. Dean, Mutat. Res., 154, 153 (1985).

3. K. Fujikawa, F. Fort, K. Samejima and Y. Sakamoto, Mutat. Res., 290, 175 (1993).

4. J. Andrade, F. Augusto and I. Jardim, Eclética química, 35, 17 (2010).

5. M.S. Garcia-Falcon, M. Perez-Lamela and J. Simal-Gandara, J. Agric. Food Chem., 52, 6897 (2004).

6. M.S. García-Falcon, B. Cancho-Grande and J. Simal-Gándara, Food Chem., 90, 643 (2005).

7. M.S. García-Falcón and J. Simal-Gándara, Food Addit. Contam., 22, 1 (2005).

8. L. Rey-Salgueiro, E. Martínez-Carballo, M.S. García-Falcón and J. Simal-Gándara, Food Chem., 108, 347 (2008).

9. L. Rey-Salgueiro, M.S. García-Falcón, E. Martínez-Carballo and J. Simal-Gándara, Food Chem., 108, 607 (2008).

10. L. Rey-Salgueiro, E. Martínez-Carballo, M.S. García-Falcón, C. González-Barreiro and J. Simal-Gándara, Food Chem., 115, 814 (2009).

11. L. Rey-Salgueiro, X. Pontevedra-Pombal, M. Álvarez-Casas, E. Martínez-Carballo, M.S. García-Falcón and J. Simal-Gándara, J. Chromatogr. A, 1216, 5235 (2009).

12. L. Rey-Salgueiro, E. Martínez-Carballo, M.S. García-Falcón and J. Simal-Gándara, Food Res. Int., 42, 983 (2009).

13. M.S. García-Falcon, B. Soto-González and J. Simal-Gándara, Environ. Sci. Technol., 40, 759 (2006).

14. C.-I. Chen and R. Taylor, Appl. Microbiol. Biotechnol., 48, 121 (1997).

15. J.I. Kilbane II, Water Air Soil Pollut., 104, 285 (1998).

16. S.M. Yakout, A.A.M. Daifullah, Desalinat. Water Treat., 52, 4977 (2014).

17. S.M. Yakout, A.A.M. Daifullah and S.A. El-Reefy, Adsorp. Sci. Technol., 31, 293 (2013).

18. S.M. Yakout and A.A.M. Daifullah, Desalinat. Water Treat., 51, 6711 (2013).

19. M. Alkan, Ö. Demirbas and M. Dogan, Micropor. Mesopor. Mater., 101, 388 (2007).

20. C. Moreno-Castilla, Carbon, 42, 83 (2004)

21. N.P. Cheremisinoff, Handbook of Water and Wastewater Treatment Technologies, Butterworth-Heinemann, Woburn, MA, USA (2002).

22. F. Cecen and O. Aktas, Activated Carbon for Water and Wastewater Treatment: Integration of Adsorption and Biological Treatment, WileyVCH; edn 1 (2011). 